\title{
5-Hydroxyindoleacetic Acid Measurement
}

National Cancer Institute

\section{Source}

National Cancer Institute. 5-Hydroxyindoleacetic Acid Measurement. NCI Thesaurus.

Code C112217.

The determination of the amount of 5-hydroxyindoleacetic acid present in a sample. 